\title{
Alcohol Use and Mental Health Status Among University Students
}

\section{in Greece}

\author{
Christos Tsiongas ${ }^{1}$, Christos Zilidis ${ }^{2}$, Evangelos C. Fradelos ${ }^{3}$, Konstantinos Tsaras ${ }^{4}$, Dimitrios
} Papagiannis $^{5}$, Ioanna V. Papathanasiou ${ }^{6}$

1. Nursing Department, TEI of Thessaly, GREECE.

2. Department of Medical Laboratories, TEI of Thessaly, GREECE.

3. Psychiatric Department Athens General Hospital for Thoracic Diseases "Sotiria", Athens, GREECE.

4. Nursing Department, TEI of Thessaly, GREECE.

5. Department of Medical Laboratories, TEI of Thessaly, GREECE.

6. Nursing Department, TEI of Thessaly, GREECE.

Introduction \& Purpose: Alcohol abuse is a common incident in college student's communities. The purpose of this research study was to evaluate the rates of alcohol use among university students in Greece and to search if there is a relation between alcohol use disorders and mental health status (depression, anxiety, stress).

Material \& Method: A cross-sectional study was contacted, in which 200 students of the Applied University of Thessaly (TEI of Thessaly) participated $(n=200)$. Students had to answer questions related to demographics and socio-economics features, and about their health behaviors and their parents' health behaviors also. For measuring the alcohol use between students the AUDIT questionnaire was used and in order to estimate their mental health status the DASS-21 questionnaire was used. After the collection and the evaluation of the questionnaires, the final sample was conclusive to 171 students $(n=171)$. Descriptive statistics such as frequencies, means, percentages and standard deviations have been utilized. Inferential statistics such as pearson $r$ correlation and regression analysis have been used to determine correlations between relevant variables. Level of significance accepted was $\mathrm{p}<0.05$.

Results: From the total of the 171 students, 75 were males $(43,9 \%)$ and 96 were females $(56,1 \%)$, with the average age of 21,15 years old (st. dev. $\pm 3,77$ ). Inductive statistics indicate relation between alcohol use and socioeconomics features of individual's parents, as well as a relation with personal health behaviors (tobacco smoking $p=0,000$ and illicit substance use $p=0,000$ ). Correlation analysis also showed statistically significance between alcohol use and mental health status is present $(p=0,000)$.

Conclusion: There are socio-economics factors and health behaviors that can influence alcohol use among university students. Alcohol use is related with mental health state in university students. However, it is not clear yet if alcohol use affects mental health or the exact opposite.

Keywords: university students, alcohol use, mental health status 